\title{
Antimicrobial peptides: To membranes and beyond
}

5 Departamento de Ciencia de los Alimentos, Instituto de Agroquímica y

6 Tecnología de Alimentos (IATA), CSIC, Apartado de Correos 73, Burjassot,

$7 \quad 46100$ Valencia, Spain

8

$9 \quad$ * Corresponding author:

10

Dr. Jose F. Marcos

Instituto de Agroquímica y Tecnología de Alimentos (IATA).

Apartado de Correos 73. Burjassot. E-46100 Valencia. Spain.

13

e-mail: jmarcos@iata.csic.es

14

Tel:

34-96-3900022

15

Fax:

34-96-3636301

16 


\section{1. Abstract}

3 Background: Antimicrobial peptides (AMP) are widely recognized as promising

4 alternatives to the current use of antibiotics and fungicides. Amino acid

5 sequences of a vast majority of AMP share cationic and amphipathic

6 biophysical properties that allow their insertion into lipid bilayers, and can lead

7 to alteration of biological membrane functions. Initial characterization studies

8 linked these properties to antimicrobial killing activity. However, additional data

9 indicate that this is not the sole mode of action and that more subtle

10 mechanisms might mediate the interaction with and effect to target microbes, as

11 well as the specificity and toxicity of peptides. As such, antimicrobial peptides

12 are increasingly viewed as powerful multifunctional drugs.

14 Objective: This review will summarize findings on these alternative non-lytic

15 modes of antimicrobial action that go beyond membrane disruption, with an

16 emphasis on the specific interaction with microbial cell wall/membrane

17 components, signaling of AMP exposure, and intracellular targets of peptide

18 action. We will also explore how novel technologies can help to reveal,

19 characterize and exploit these antimicrobial properties.

21 Conclusion: Detailed knowledge on non-lytic modes of action of antimicrobial

22 peptides will help in the design and discovery of novel antibacterial and

23 antifungal compounds. 
1 Keywords: Antibacterial peptide, antifungal peptide, antimicrobial mechanism,

2 apoptosis, cell-penetrating peptides, chaperone, glycan, MAPK signaling,

3 membrane permeation, two-component system. 
3 Antimicrobial peptides and/or small antimicrobial proteins (AMP) have been

4 characterized from a vast number of organisms, from bacteria to insects, plants

5 and humans [1]. The increasing problem of antibiotic resistance in clinic [2,3]

6 and the pressure to reduce antibiotic and fungicide use in agricultural and food

7 industry [4-7] has put AMP at the edge front as promising compounds to fight

8 microbial infections and contaminations. Over 1000 natural AMP are currently

9 known to their amino acid sequence. Moreover, the advent of amenable

10 synthetic procedures, wide offer of biotech companies with peptide synthesis

11 facilities, and high throughput (HT) approaches to synthesize and screen large

12 collections and libraries of peptides have increased substantially the number

13 and diversity of non-natural synthetic peptides with antimicrobial activity. Thus

14 an enormous amount of peptides endowed with antimicrobial activity are

15 currently known. However, only a minor proportion of them have been

16 characterized in detail and studied in relation to their antimicrobial mode of

17 action.

19 AMP share common biophysical properties. They are small, from just 5-6 amino

20 acid residues in some synthetic peptides to about 50 -and even up to 100- in

21 natural ones; most of them (although not all) are cationic having positive charge

22 at physiological conditions due to the presence of arginine and lysine residues,

23 also have a high proportion (up to $50 \%$ ) of hydrophobic residues, and are

24 capable to fold or arrange into a variety of amphipathic structures and

25 conformations. Cationic charge and amphipathic arrangement are on the basis 
1 of their propensity of in vitro interaction with anionic lipid bilayers. In fact, initial

2 studies after discovery of the first AMP were coincident in concluding that

3 antimicrobial activity was a primary consequence of the capacity of cationic

4 amphipathic peptides to interact and disrupt biological membranes, thus

5 resulting in direct cell killing. Treatment of microorganisms with above minimal

6 inhibitory AMP concentration resulted in microbial cell permeation that

7 correlated with microbicidal potency. As consequence, many of the

8 contributions that dealt with AMP mechanism relied on models of peptide

9 interaction with -and disruption of- lipid bilayers.

11 However, a key weakness point was that AMP cationic charge led to the early

12 recognition that salts -at concentrations close to physiological- blocked AMP

13 interaction with negatively charged microbe surface groups, and also

14 diminished the in vitro inhibitory activity of microorganism growth. It was difficult

15 to reconciliate this apparent limitation with the evidence that the high number

16 and diversity of AMP in living organisms is indicative of an important

17 physiological role, presumably in host defense.

19 A way out of this dilemma derives from the recognition of multilayer roles of

20 peptides in the regulation of host response, also acting on specific cells as

21 effectors of the adaptive immune system [8]. Representative examples are

22 human defensins and the cathelicidin LL-37 [9,10]. In particular, human

23 defensins have emerged as an evolutionary link that bridges innate and

24 adaptive immune responses [10]. But there are also additional alternatives to

25 justify the prevalence of AMP in nature. 
2 If specific AMP can participate in complex processes such as regulation of the

3 mammal immune response, it is not senseless that can also exert multifaceted

$4 \quad$ lethal actions on microbes. In fact, alternative mechanisms have been

5 increasingly considered as part of AMP action against microbes [11-13].

6 Reports have analyzed peptide action towards microbes (not under in vitro

7 membrane mimicking lipid environments) in greater detail, and accumulated

8 convincing evidence that specific peptides do not exert antimicrobial activity by

9 primarily permeabilizing cell membranes. Among these are apidaecin [14] or

10 dermicidin [15] acting on bacterial cells, and specific human defensins and

11 histatin-5 $[16,17]$ or synthetic peptides $[18,19]$ on fungal cells. Therefore,

12 alternative non-lytic modes of action are recognized. Recent reviews have

13 addressed this question in the case of antibacterial peptides $[20,21]$ or the

14 antifungal action of defensins [22]. This contribution aims to provide an updated

15 and broader view to these alternative AMP modes of action or interactions with

16 microbes, to the approaches to further investigate the extent and significance of

17 these, as well as to discuss how this knowledge can be incorporated to the

18 design of novel and improved AMP with higher antimicrobial potency and lower

19 unspecific toxicity. The use of (model) microorganisms for which genomic-scale

20 tools are available and the identification of genes that modulate the microbial

21 susceptibility will be pivotal in the understanding of peptide antimicrobial action.

22 We will not address, however, the responses of microbes to counteract peptide

23 action, that recent reviews summarize in detail [23].

25 3. Interaction with microbial surface. 
2 Before reaching cell membranes, the first layer of contact for surrounding

3 peptides is the outer microbial surface. There was no evidence of specific and

4 canonical receptors linked to the interaction of peptides with target

5 microorganism and microbial killing. Early reports on the similar activity of

6 stereoisomeric AMP sustained the conclusion that interaction was not stereo-

7 specific [24]. Recently, non-chiral interaction has even been shown for peptides

8 acting intracellularly [25]. However, there were also examples for which

9 stereospecificity was shown [26], and in some cases correlated with an

10 antimicrobial mechanism that was not pore-forming [14].

12 Several AMP bind bacterial lipopolysaccharide (LPS), and such binding is linked

13 with the differential antimicrobial activity of peptide analogs with lower affinity for

14 LPS $[27,28]$ (Table 1). On the cell side, changes in cell wall/lipid bilayer

15 composition can alter interaction and thus activity of peptides. A mechanism of

16 bacterial resistance to peptide exposure involves the alteration of cell

17 envelope/membrane, to increase the net charge thus reducing the electrostatic

18 interaction with cationic antimicrobial peptides [23,29-31] (Table 2). More

19 intriguingly, biophysical properties of specific synthetic peptides [32], or distinct

20 combinations of temporins acting synergistically [33] can indeed modulate the

21 translocation of peptides across bacterial outer envelopes and allow their

22 access to and interaction with bacterial membranes.

24 In addition to electrostatic attraction, specific cell membrane/wall components

25 have been shown to promote the interaction with peptides. A significant 
example is the antibacterial peptidic lantibiotic nisin, for which it is established

2 that the membrane-bound peptidoglycan precursor Lipid II acts as a docking

3 moiety to attract the peptide to the bacterial membrane and promote peptide

4 insertion into membrane and cell permeation [34-36]. Interestingly, nisin is

5 active at nanomolar range against bacteria containing Lipid II, roughly three

6 orders of magnitude more active than peptides that only act through

7 permeation. Nisin is not active against yeast or filamentous fungi; however,

8 yeast spheroplasts are rapidly lysed when incubated in the presence of nisin at

9 concentrations which do not affect intact cells [37]. This latter activity is

10 presumed to be a consequence of the intrinsic capacity of nisin to disturb lipid

11 bilayers. Thus, even in the case of true membrane-perturbing peptides,

12 additional factors such as their propensity to interact with specific compounds

13 might modulate (and enhance) their (membrane) activity. Interestingly, the

14 related peptidic lantibiotic mersacidin also binds Lipid II, albeit at its terminal N-

15 acetylglucosamine, and inhibits bacterial growth with no cell permeation

$16[38,39]$. Because of these binding capacities nisin, as well as mersacidin, also

17 interfere with the peptidoglycan biosynthesis of bacterial envelope [35] (Table

18 1), being one clear example of multilayer actions among AMP.

20 Nisin is a well known example, but analogous situations may occur with specific

21 defensins, whose antifungal activity is linked to the presence of distinct classes

22 of membrane glycolipids. Hence, the activity of the plant defensin DmAMP1 and

23 the cyclic lipopeptide syringomycin E are dependent on the biosynthesis of the

24 acidic sphingolipid mannosyl diinositolphosphoryl ceramide, since the presence

25 of functional IPT1 and SKN1 genes were linked to an enhanced resistance 
1 phenotype of the yeast S. cerevisiae [40-42] (see Table 1 and Table 2). Also,

2 the plant RsAFP2 or the insect heliomycin are coincident in requiring the

3 presence of the neutral sphingolipid glucosyl ceramide $[43,44]$. The $F$.

4 graminearum GCS1 gene involved in the biosynthesis of glucosyl ceramide also

5 mediates the susceptibility to the plant MsDef1 defensin but, interestingly, not to

6 the related MtDef4 [43]. It has been postulated that sphingolipid-rich lipid rafts

7 could promote interaction with membranes of a subclass of defensin AMP [22].

8 However, other related defensins do not specifically require such membrane

9 lipids, and in fact additional defensin-interacting molecules have been

10 demonstrated [45]. On the other hand, plant PSD1 seems to operate through a

11 distinct mechanism since it has been shown to be internalized and act on

12 intracellular targets (see below) [46]. Therefore, although structurally related,

13 the broad class of defensin peptides do comprise AMP with distinct interacting

14 partners and effects on target cells.

16 A repeated issue in several of these examples is that sugar moieties (mostly as

17 part of more complex molecules) seem to act as interacting/docking partners for

18 a variety of distinct AMP. In addition to the examples described above,

19 phosphomannans of yeast cell wall mannoproteins increase toxicity of the

20 antifungal osmotin, probably by serving also as docking structures that facilitate

21 the interaction and diffusion across the cell wall [47] and numerous antifungal

22 proteins are known for their ability to bind chitin $[48,49]$. Mutants of filamentous

23 fungi with specific chitin synthase genes disrupted show alterations in their

24 sensitivity to antifungal proteins [48] (Table 2). In this regard, specific cell wall

25 components, including glycans and glycoproteins, have been recognized as 
1 potential targets for selective antifungals [50-54]. It would be desirable the

2 detailed characterization of the interaction of selected AMP with such glycan

3 structures, in order to obtain information on both the potential microbial targets

4 and the peptide structural requirements for activity.

6 Additional studies report on the involvement of cell wall proteins on the fungal

7 susceptibility to AMP. In several examples, the relevance of specific cell wall

8 proteins is likely due to a general strengthening of cell wall that increases

9 thickness or reinforces its structural resistance after exposure to peptides, as is

10 the case of yeast PIR proteins in the interaction with tobacco osmotin [55] or

11 other CWP proteins with nisin [37].

13 A remarkable example studied in detail is that of SSA1 and SSA2, cell surface

14 proteins from Candida that mediate the activity of distinct AMP as human $\beta$ -

15 defensins and histatin, but not of human neutrophil defensins $[45,56]$. These

16 proteins are highly conserved in organism from distinct phylogenetic scale as

17 part of the ATPase heat shock protein 70 (HSP70) family. In Saccharomyces,

18 they have been localized to the nucleus, cytoplasm and cell wall, and are

19 chaperones pivotal in active protein refolding as response to stress. Binding of

20 histatin-5 to Candida cell wall SSA1/2 proteins was demonstrated and also

21 linked to fungicidal activity [56,57]. This binding is necessary but not sufficient

22 for the cell killing activity of the peptide [57], which also requires peptide cell

23 internalization (see below). Histatin-5 binding has been recently mapped to the

24 ATPase domain of SSA2 protein and showed to be enhanced by protein-bound 25 nucleotides [58]. 
2 Finally, there are peptides whose effect is expressed at the microbial

3 surface/membrane but are known as non-lytic. The synthetic BM0 was identified

4 as an inhibitor of the activity of a yeast plasma membrane ATPase (Pma1) that

5 impairs in vitro growth with no permeation at growth-inhibitory concentrations

6 [18]. The peptide locates and is reported to be active at the cell surface.

8 4. Signaling of peptide exposure

10 An antimicrobial action solely based on permeation is expected to be quick, not

11 allowing a coordinated cell response to peptide exposure. However, a variety of

12 different observations indicate that microorganism respond to antimicrobial

13 peptides in different ways. For instance, transcriptome analyses have shown

14 global changes in gene expression after exposure to distinct AMP in both

15 bacteria [59-61] and fungi [62-64] (see below). These studies underline a

16 response of microorganism that might be related to peptide mechanism of

17 antimicrobial action and/or be part of the mechanisms to counteract peptide

18 deleterious effect. In any case, some of these reports also indicate the

19 existence of cell signaling components that coordinate such responses.

21 In bacteria, two-component and related sensor systems participate in AMP

22 recognition and induction of transduction cascades that modulate the bacterial

23 response to peptide exposure $[59,65]$. The two-component system PhoPQ is a

24 determinant of virulence in a number of Gram-negative bacteria and mediates

25 the interaction with AMP $[30,66,67]$, and the membrane bound sensor kinase 
1 PhoQ is activated by cationic AMP binding to an acidic surface domain [65].

2 The additional PmrAB two-component system also responds and mediates the

3 response and resistance to AMP $[61,68,69]$. An analogous three-component

4 system could operate in Gram-positives and is involved in the signaling and

5 coordinated expression of different responses to AMP [59]. These sensor

6 systems are part of the bacterial defensive armor and a general response

7 mediated by them is the modification of the anionic charge of the bacterial

8 surface to reduce peptide interaction (see below). Therefore, they would not be

9 directly linked with the killing mechanism of AMP. However, the detailed

10 knowledge of their involvement in the response to peptides and the structural

11 characterization of the interaction could lead to the design of AMP capable of

12 by-passing or disturbing these bacterial surveillance systems.

14 In yeast, a transmembrane receptor-like protein is required for sensitivity to

15 osmotin, and functions upstream of RAS2 in a signaling pathway that induces

16 apoptosis after exposure to the AMP $[70,71]$. Different fungal protein kinase

17 signaling cascades mediate the response to distinct antimicrobial peptides and

18 proteins, and mutations in the corresponding genes resulted in increased

19 sensitivity $[63,72,73]$ (Table 2). At least in some examples, the involvement of

20 each pathway seems to be dependent of the specific peptide as was nicely

21 demonstrated in the case of two related plant defensins [73]. The increased

22 sensitivity of fungal cells deleted in components of these signaling cascades is

23 indicative that they are part of the microbial response to peptide exposure and

24 damage, and not necessarily linked to the peptide antimicrobial action. 
1 On the other hand, independent studies indicate that specific AMP (such as frog

2 dermaseptins, human lactoferrin, tobacco osmotin or fungal AFP) induce

3 apoptosis markers in yeast [71,74,75] or filamentous fungi [76]. Contrary to the

4 above discussed data, mutation of involved genes enhance resistance to

5 peptides, which supports that induced microbial suicide is indeed part of the

6 fungal killer mechanism in these examples [71,74,76]. Reactive oxygen species

7 (ROS) are known as markers of cell suicide [77], and additional studies broaden

8 the number of AMP for which their effect is associated with intracellular ROS

9 production, which also suggest an induction of intracellular signaling pathways

$10[71,78,79]$. However, the role of ROS in antimicrobial action remains

11 controversial for specific peptides such as histatin [80,81].

13 5. Cell internalization

15 Distinct AMP have been demonstrated to translocate across the cell membrane 16 in a non-disruptive mechanism, and examples exist in bacteria and fungi. Insect 17 apidaecin was one of the first AMP for which a non-pore forming mechanism

18 was invoked [14], and early uptake assays with radiolabeled peptide indicated

19 retention by $E$. coli cells [82]. The use of fluorescently labeled peptides and

20 proteins coupled to confocal microscopy has boosted the demonstration of cell

21 internalization of this and other AMP [19,46,83-89]. Additional techniques such

22 as immunodetection were used to show that antimicrobial proteins enter fungal

23 hyphae [87]. In several of these reports, peptide internalization was shown at

24 peptide concentrations and/or times of exposure at which no obvious growth

25 alteration or cell membrane damage could be observed. For instance, the 
1 synthetic hexapeptide PAF26 was internalized at sub-inhibitory concentrations

2 by fungal hyphae and caused severe morphological alterations in the absence

3 of mycelium permeation, which was finally produced at higher completely

4 inhibitory concentrations [19]. Another noteworthy example is that of the

5 synthetic NK-2 peptide, which is selectively internalized by Plasmodium infected

6 red blood cells due to the increase in the negative charge of the membrane that

$7 \quad$ occurs in infected cells, and subsequently kills the protozoan pathogen

8 intracellulary [90].

10 Targeting of peptides to specific cell compartments has also been shown and in 11 some cases linked to the antimicrobial activity. Thus, the pea defensin PSD1

12 was shown to locate inside the nucleus of the model fungus Neurospora crassa,

13 wherein it might alter cell cycle progression [46]. Also, histatin-5 at low (inactive)

14 concentrations is directed towards yeast vacuoles, while inhibitory higher

15 concentrations locate in the cytosol [17].

17 A paradigmatic example of cell penetration is that of antimicrobial peptides

18 derived from bovine lactoferrin, which have been shown to be internalized by

19 both bacterial and yeast cells $[86,91]$. It remains to be determined, however,

20 whether cell internalization share common mechanism for both classes of

21 microorganisms.

23 The capability to penetrate target cells is therefore increasingly viewed as a

24 common property of distinct cationic AMP (Table 1). AMP having cell

25 translocation activity share biophysical properties with the so-called cell 
1 penetrating peptides (CPP), which has brought into question the differences

2 between antimicrobial and cell penetrating peptides [92]. CPP have been

3 proposed as shuttle vehicles for the delivery of killing or therapeutical molecules

4 into (mammalian) cells [93]. Peptides initially known as CPP onto mammalian

5 cells have been later demonstrated to have antimicrobial effects on distinct

6 microorganisms, and this activity correlated with internalization into microbial

7 cells [94,95]. An attractive hypothesis is therefore that this class of peptides are

8 in fact dual molecules in which internalization determinants do not necessarily

9 overlap with antimicrobial [21]. Interestingly, for selected AMP such as the

10 proline-rich apidaecin the microbial internalization and killing activities seem to

11 be separated, and peptide uptake was necessary but not sufficient for

12 antibacterial activity of selected analogs [82]. Experiments should address the

13 identification of these (separate) determinants in additional model AMP, in order

14 to determine the minimum amino acid sequence requirements for these

15 activities, if any, and help in the design of "modular" domains with distinct

16 functional capabilities.

18 An still unsolved question relates to the actual mechanism(s) of non-lytic

19 peptide internalization [96,97]. Despite numerous efforts, the underlying

20 mechanism of AMP/CPP uptake is still unclear and controversy exists, which

21 likely reflects the involvement of distinct pathways and processes depending on

22 the peptide and cells under study. Several peptides have been demonstrated to

23 be internalized in an active, energy-dependent process $[82,88]$. Internalization

24 may follow multiple and simultaneous endocytic pathways, and even at high

25 peptide concentrations a non-lytic endocytosis-independent uptake [97]. Initial 
1 interaction seems to depend on cell surface complex glycans [98]. Deciphering

2 the mechanism of peptide cell penetration including the related

3 similarities/differences among CPP acting on mammalian cells and AMP on

4 microbes, will undoubtedly help to design improved antimicrobial peptides

5 endowed with higher specificity.

6

7 AMP sequence analogs will surely help to characterize the above described

8 open questions such as the internalization mechanism/pathways, and

9 determine structure activity relationships [84]. An study showed the importance

10 of a single proline residue at the hinge region of buforin, required for bacterial

11 membrane translocation [83]. This residue is also critical for maintaining both

12 antibacterial and antifungal activities of the peptide. Interestingly, buforin

13 analogs with the proline substituted did not penetrate bacterial cells but rather

14 remained at the surface and seemed to kill bacteria through permeation.

16 6. Intracellular targets

18 It is expected that AMP that translocate into cells might disturb cell homeostasis

19 in different ways determined by their intrinsic properties as well as their

20 targeting/interaction with cellular organelles. Due to their cationic nature, most

21 AMP readily bind nucleic acids (DNA and RNA) in vitro which might result in a

22 broad inhibition of DNA synthesis, transcription and/or mRNA translation inside

23 cells [82,85,99-101]. Even for some peptides known to cause permeation, an

24 inhibition of DNA, RNA and/or protein synthesis was reported [102,103],

25 indicating that disruption of cell membranes might be combined with inhibition of 
1 intracellular targets. However, it is obvious for nearly all the examples analyzed,

2 that nucleic acid binding by known AMP is quite unspecific, at least in vitro.

3 Therefore, specificity of inhibition of such peptides would be derived from the

$4 \quad$ interaction with outer microbial envelopes or cell membrane components that

5 enable internalization.

6

$7 \quad$ More specific intracellular mechanisms have also been proposed. Distinct

8 approaches have been used to identify host protein partners of AMP. Affinity

9 purifications identified S. cerevisiae DNA binding proteins involved in DNA

10 repair, as partners of the AMP dermaseptin S3 [74], and contributed to the

11 understanding that its mode of action is related to the induction of apoptosis in

12 yeast. Similarly, immunoaffinity purification of proline-rich AMP incubated with

13 E. coli proteins identified the DnaK and GroEL bacterial chaperones with the

14 ability to bind AMP, and DnaK binding was shown to be related to bacterial

15 killing $[104,105]$ (Table 1). DnaK is an ATPase HSP that is similar to the class of

16 SSA proteins from yeast that bind to histatin (see above), and in fact the AMP

17 pyrrhocoricin binding inhibits ATPase activity and protein refolding by the

18 chaperone, which would led to accumulation of misfolded proteins and lethality

19 [104]. DnaK and related chaperones are also overexpressed in Lactococcus

20 lactis strains with enhanced resistance to nisin [106]. These chaperones are

21 biologically selected for recognition and binding of aberrantly folded

22 proteins/peptides, and as such are critical for the cell response and survival to

23 various types of stress (for instance, heat stress). Therefore, it seems that

24 distinct classes of AMP are prone to interact with specific cell chaperones. It 
1 remains to be determined whether this is a behavior that extends to other AMP,

2 and how this is related to cell killing.

4 A unique example of intracellular target is that of the pea defensin PSD1, which

5 in a yeast two hybrid approach interacted with several fungal nuclear proteins,

6 including cyclin F with which also in vitro binding was demonstrated [46]. The

7 defensin translocated to the fungal nucleus (see above) and further analysis

8 indicated that affects normal cell cycle progression.

10 Several members of the mammalian superfamily A of RNases have been

11 shown to have a direct antimicrobial function, and be involved in the innate

12 immune system [107]. Plant antimicrobial PR-10 proteins also have

13 ribonuclease-like properties. The peanut RNase AhPR-10 has been shown to

14 locate inside hyphae and kill susceptible fungi [108]. Interestingly, a point

15 mutation devoid of RNase activity internalizes into hyphae but does not inhibit

16 fungal growth or disrupt membrane permeability, thus separating the cell

17 penetration from the ribonuclease activity and, further, linking this and the

18 antimicrobial properties. Although not related with nuclease activity, there are

19 previous examples in which cell penetration has been separated from peptide

20 antimicrobial activity [82], thus confirming that penetration and killing can be

21 separated steps of the antimicrobial mechanism of peptides.

23 Also, there are examples of AMP that are not internalized although elicit an

24 intracellular action, as specific plant defensins that induce extended 
1 citosqueleton disorganization despite being retained the cell surface of yeast

2 cells [72].

4 7. Genome-wide analyses of microbial response to peptide exposure.

6 The use of genomic tools is expected to help in the characterization of

7 alternative modes of AMP action, including the effects on microorganisms, the

8 determinants of susceptibility to peptide action and the identification of potential

9 microbial targets. These approaches will lead to the identification of microbial

10 genes that modulate sensitivity to peptides (Table 2), as a critical part of the

11 detailed knowledge of AMP action. However, few studies have been reported

12 yet, so as to draw general conclusions. Most of these have been conducted by

13 exposing model organisms such as $E$. coli or $S$. cerevisiae to sub-lethal peptide

14 concentrations, and analyzing the transcriptomic response or testing the

15 susceptibility of genome-wide mutant collections. An alternative approach is to

16 compare the transcriptional profile of AMP-resistant bacterial strains with that of

17 the corresponding parentals [106].

19 These types of studies have been carried out both in Gram-negative (i.e., E.

20 coli) and Gram-positive bacteria [59-61,106]. A major transcriptional change

21 relates to stress response, and also to the attempt to reduce peptide interaction

22 and exposure, namely by increasing the net charge of the bacterial surface

23 (explained above), strengthening of exopolysaccharide envelope, and inducing

24 extrusion/transporter systems $[59,61,106,109]$. Interestingly, it was found that

25 selected E. coli genes respond differently to distinct AMP as occurs with the 
1 sensor BasS of the AMP responsive two-component BasRS [61] (see also

2 above), reflecting a specificity in AMP sensing and/or action that would depend

3 on the peptide sequence. This latter work also showed that non-active

4 sequence analogs do not exert the same transcriptional response as the

5 parental antimicrobial peptide. In any case, it is noteworthy that most of the

6 genes that respond significantly to AMP correspond to proteins of unknown

7 function $[60,61]$, implying the existence of a part of the microbial response that

8 clearly needs to be characterized in detail and might be related to the

9 activity/specificity of the peptides.

11 There is also a limited number of reports on the analysis at a genome scale of

12 the effect of AMP on fungi, and these have been focused on unicellular yeasts

13 [62-64]. Conclusions need to be critically tested for their relevance to

14 filamentous fungi. For instance, a common report in yeast is the induction by

15 distinct AMP of the osmotic stress response regulated by the HOG MAPK

16 pathway, involved in osmotic tolerance and cell wall maintenance $[62,63]$.

17 Indeed, it seems to be a cross-tolerance phenotype between AMP and osmotic

18 stress [62]. However, the HOG pathway does not participate in the response of

19 F. graminearum to specific defensins [73].

21 A direct comparison of a S. cerevisiae genome-wide screen between two

22 unrelated AMP that had been previously known to kill by permeation -a

23 dermaseptin derivative and magainin 2-indicate that their actions are more

24 complex than membrane disruption, and also demonstrated common and -

25 interestingly- unique effects of each peptide [64]. Thus, treatment with either 
1 peptide elicits responses related to DNA damage that would be part of a

2 general reaction to damage/stress. Likewise, a significant functional annotation

3 related with ribosome assembly and rRNA processing was found among genes

$4 \quad$ repressed by both peptides. However, and considering changes in gene

5 expression and alterations of susceptibility of deletant strains, only a minor

6 proportion of the nearly 5,000 genes analysed showed common behaviours

7 among the two peptides. For instance, no gene deletions were found that

8 conferred increased resistance to both peptides. Deletion of genes involved in

9 vacuolar transport and protein targeting to the vacuole increased sensitivity to

10 the dermaseptin peptide [64]. An independent work showed that over-

11 expression of a different vacuolar $\mathrm{H}+-\mathrm{ATP}$ ase increased resistance to a distinct

12 AMP [110], and targeting of histatin-5 to vacuoles from the surrounding

13 cytoplasm seems to be a survival mechanism in Candida [17]. Thus, different

14 approaches that include genomic screens identified transport to fungal vacuoles

15 as a common mechanism of defence to AMP.

17 Also, comparisons among genomic screens based on collections of mutant

18 strains or on transcriptomic data indicate that both approaches are

19 complementary and required for a deep characterization of AMP mechanisms,

20 since they do not necessarily led to the identification of the same gene sets [64].

21 An additional approach is also the screening of cDNA expression libraries to

22 identify genes whose over-expression can influence the sensitivity of fungi to

23 AMP [110], which permitted the identification of genes that led to increased

24 either resistance or susceptibility to a plant defence peptide. Authors report that

25 these would have not been identified in deletant mutant screens since the 
1 corresponding knockout strains did not show alterations of sensitivity to

2 peptides.

4 A limitation of the above studies on fungi is that they use unicellular yeasts,

5 namely S. cerevisiae. Although it has obvious advantages, S. cerevisiae is also

6 limited as a model organism. AMP active against laboratory yeast strains may

7 have no effect on pathogenic yeast or filamentous fungi and viceversa. Also, S.

8 cerevisiae has undergone gene duplication during evolution that may produce

9 function redundancy and bias the analysis of peptide antifungal activity. Despite

10 these disadvantages, the use of yeast as model for drug (antimicrobial peptide)

11 characterization and development has a wide potential, albeit similar studies

12 should be extended/confirmed onto filamentous fungi and must be encouraged.

14 A significant conclusion in all these genome-wide studies with both bacteria and

15 fungi is that genes involved in known resistances to nowadays antibiotics and

16 fungicides are not usually identified, indicating that AMP are indeed compounds

17 of novel modes of action that could aid in the development of novel

18 antimicrobial strategies.

20 8. Expert opinion

22 Researchers have agreed over the last years that non-lytic modes of AMP

23 action exist, and even co-exist with membrane disrupting properties of well

24 known lytic peptides [11,20-22]. In our view such alternative mechanisms go

25 beyond membrane interaction and in a broad sense might explain the specific 
1 properties of selected peptides, including their specificity towards certain

2 microbes or cells and also their potency not always correlated with their

3 membrane permeation capacity. As summary, Figure 1 shows a conceptual

4 model of the steps of AMP action onto microbes that includes, but also goes

5 beyond, their interaction with biological membranes. Three major steps are

6 envisioned as relevant to accommodate the current knowledge of AMP

7 mechanisms in a general model of peptide antimicrobial action: (1) Interaction

8 with outer microbial structures, (2) interaction with cell membrane that can result

9 in peptide sensing that signals peptide exposure, internalization/translocation to

10 cell interior, and/or disruption of lipid bilayer, and (3) intracellular targets that are

11 related to/explain peptide activity. In this review we have discussed examples

12 on how specific the activity of selected peptides can be, regarding all these

13 steps. Previously not foreseen examples are the significance of stress-related

14 chaperones observed with distinct microorganisms and peptides at the level of

15 protein:peptide interactions [57,58,105], inhibition of activity [104], and

16 responsive genes [106]. Also, the importance of vacuolar transport and peptide

17 targeting to the vacuole as suggested by overexpression/deletion of related

18 genes [64,110], and localization data [17]. Detailed knowledge of the molecular

19 and cellular bases of each one of these steps in an increasing number of

20 peptides/microbes (including clinical and agronomically relevant pathogens)

21 might allow the design of AMP with increased potency and lower unspecific

22 toxicity.

24 In our view, studies on antimicrobial peptide action must be shifted from the 25 peptide structural requirements/biophysical properties to the cell determinants 
1 of sensitivity to peptides. The use of model organisms, genomic approaches,

2 and screening/selection of collections of mutants will be pivotal to unravel the

3 mechanism of action of selected peptides. We have given some relevant

4 examples of this $[61,64,106]$, but they are still scarce. These types of studies

5 are identifying relevant genes modulating sensitivity to AMP (Table 2). With an

6 increasing number of reports we will be in good position to ask the fundamental

7 question on whether there are non-lytic modes of AMP action "common" to

8 distinct peptides and whether each peptide class has specific properties not

9 shared by others. Also related, we will be able to classify AMP in relation to their

10 effect on target cells, as well as to identify those cell targets that are more

11 promising in terms of potency and specificity.

13 The use of synthetic peptides and their sequence analogs will be critical in

14 establishing structure/activity relationships between peptide sequence,

15 antimicrobial activity and effects on microorganisms, with the potential for

16 extrapolation to the genetic/molecular determinants of peptide susceptibility of

17 the latter. It is intriguing, for instance, that highly related plant defensins seem to

18 interact with distinct cell components/signaling cascades [43,73]. A

19 consequence is that minor amino acid changes in AMP could be responsible for

20 differences in the specific modes of action [35,57]. Due to their small size and

21 feasibility of synthetic production, small AMP can be used to dissect the

22 molecular basis of such differences and specificities.

24 It is conceivable that an holistic combination of detailed knowledge of modes of 25 action, genome screens, high-throughput peptide identification technologies, 
1 and amino acid sequence requirements, could lead to the development of novel

2 rationally designed AMP to be used as drugs against functionally important

3 microbial targets.

4

5 Among the very interesting potential of AMP, the elucidation of specific modes

6 of action constrained in relatively small peptide molecules might allow the

7 combination of more than one killing mechanisms in one single AMP, as

8 multitarget drugs, and could potentiate the antimicrobial activity while

9 diminishing the probability of developing resistance in the susceptible microbes

$10 \quad[35]$.

11

129. Acknowledgements

13

14 We apologize to all the investigators whose research could not be appropriately

15 cited due to space limitations. Our work on antimicrobial peptides has been

16 supported by grants $\mathrm{BIO} 2003-00927$ and BIO2006-09523 (Spain). M.G. is

17 recipient of a research contract from the JAE-DOC Program (CSIC, Spain). 
$1 \quad$ Figure Legend

3 Figure 1.

4 General model of the antimicrobial mechanism of AMP. The figure shows a

5 schematic view of an eukaryotic cell, although the conceptual framework can be

6 also applied to bacterial cells. A major difference would rely on peptide import to

7 nucleus, which has been demonstrated for specific AMP in fungal cells (see text

8 for details). Three major steps are depicted in the antimicrobial mechanism of

9 AMP (blue line): (1) Interaction with outer microbial structures, (2) interaction

10 with cell membrane components that results in disruption of lipid bilayer/pore-

11 formation (2a), internalization/translocation to cell interior (2b), and/or signaling

12 of peptide exposure (2c); and (3) intracellular targets (as detailed, $3 a-3 d$ ) that

13 have been demonstrated in distinct examples as related to peptide activity (see

14 text for details). The relevance of glycan structures for peptide interaction is

15 highlighted by hexagons within cell wall/envelope glycans, membrane lipids,

16 and glycoproteins. AMP folding (thick blue line) may change upon each step. 
Table 1. Selected examples of non-lytic antimicrobial peptide mechanisms

\begin{tabular}{|c|c|c|c|c|c|}
\hline Peptide & Microorganism & Interaction/Binding & Cell Uptake & Mechanism & References \\
\hline Nisin & Bacteria & Lipid II & & Inhibition of peptidoglycan synthesis & {$[35,36]$} \\
\hline Buforin II & Bacteria & & Yes & Binding DNA/RNA & {$[83,85]$} \\
\hline Magainin 2 & Bacteria & LPS & Yes & & {$[91,111]$} \\
\hline Indolicidin & Bacteria & LPS & Yes & $\begin{array}{l}\text { Binding to nucleic acids/Inhibition of } \\
\text { proteins and nucleic acid synthesis }\end{array}$ & {$[99,112]$} \\
\hline Pyrrhocoricin & Bacteria & & Yes & $\begin{array}{l}\text { HSP (DnaK) binding / Prevention of } \\
\text { chaperone protein folding }\end{array}$ & {$[84,104]$} \\
\hline Lactoferricin B & Bacteria & LPS & Yes & $\begin{array}{l}\text { Binding to nucleic acids / Inhibition } \\
\text { of macromolecular synthesis }\end{array}$ & {$[28,91,113]$} \\
\hline DmAMP1 & Fungi/yeast & $\mathrm{M}(\mathrm{IP}) 2 \mathrm{C}$ sphingolipid & & & [42] \\
\hline RsAFP2 & Fungi/yeast & GlcCer sphingolipid & & ROS & [79] \\
\hline Psd1 & Fungi & & Yes & $\begin{array}{l}\text { Binding to Cyclin F / Cell cycle } \\
\text { impairment }\end{array}$ & {$[46]$} \\
\hline Osmotin PR5 & Yeast & $\begin{array}{l}\text { Membrane Receptors / } \\
\text { Phosphomannoproteins }\end{array}$ & & Apoptosis & {$[47,70]$} \\
\hline BM0 & Yeast & & No & $\begin{array}{l}\text { Inhibition of Plasma membrane } \mathrm{H+-} \\
\text { ATPase }\end{array}$ & {$[18]$} \\
\hline Dermaseptin S3 & Yeast & & & Apoptosis, ROS and DNA damage & [74] \\
\hline Histatin-5 & Yeast & SSA $1 / 2$ proteins & Yes & ROS (i?), Subcellular targeting & {$[17,58,80]$} \\
\hline
\end{tabular}


Table 2. Representative examples of genes that modulate the sensitivity of microorganisms to antimicrobial peptides.

\begin{tabular}{|c|c|c|c|c|}
\hline Peptide & Microorganism & Genes & Functional annotation/category & References \\
\hline Different AMP & S. typhimurium & pagP & Lipid A acylation & {$[30]$} \\
\hline Different AMP & S. aureus & Dlt Operon & D-alanine esterification of LTA & [29] \\
\hline Different AMP & S. aureus & MprF & Lys modification of PG & [31] \\
\hline \multirow[t]{2}{*}{ Nisin } & L. lactis & DItD & D-alanine esterification of LTA & [106] \\
\hline & & AhrC & $\begin{array}{l}\text { Transcriptional repressor of Arc } \\
\text { operon (Arginine catabolism) }\end{array}$ & [106] \\
\hline \multirow[t]{3}{*}{ Protamine } & S. meliloti & ExoT, ExoU, NdvB & $\begin{array}{l}\text { Exopolysaccharide and glucan } \\
\text { biosynthesis }\end{array}$ & [109] \\
\hline & & Y01361, HutU, UreF & Nitrogen metabolism & [109] \\
\hline & & Y01826 & $\mathrm{ABC}$ membrane transporter & [109] \\
\hline \multirow[t]{2}{*}{ DmAMP1 } & S. cerevisiae & IPT1 & Sphingolipid biosynthesis & [42] \\
\hline & & SKN1 & Sphingolipid biosynthesis & {$[40]$} \\
\hline Syringomicyn E & S. cerevisiae & IPT1, ELO2, ELO3, CSG1, CSG2 & Sphingolipid biosynthesis & [41] \\
\hline \multirow[t]{2}{*}{ MiAMP1 } & S. cerevisiae & YGL191W & Mitochondrial Cyt. c ox. sub. & [110] \\
\hline & & VMA11 & Vacuolar $\mathrm{H}^{+}$-ATPase sub. c & [110] \\
\hline
\end{tabular}


PTC7

Protein phosphatase type 2C

\begin{tabular}{lllll}
\hline Dermaseptin S3 & S. cerevisiae & Izh2, Izh3, Stm1, Aif1 & Regulation of Apoptosis & [74] \\
\hline $\begin{array}{l}\text { MsDef1 } \\
\text { MsDef1 / MtDef2 }\end{array}$ & F. graminearum & GCS1 & Glucosylceramide synthase & [43] \\
I RsAFP2 & F. graminearum & STE11, STE7, GPMK1, MGV1 & MAPK signaling cascade & [73] \\
AFP & A. oryzae & ChsB, CsmA & Chitin synthases, classes III and V & [48] \\
\hline AFP & F. oxysporum & ChsV & Chitin synthase, class V & [48] \\
\hline
\end{tabular}


1. Zasloff M. Antimicrobial peptides of multicellular organisms. Nature 2002;415:389-395

2. Hancock REW, Sahl HG. Antimicrobial and host-defense peptides as new anti-infective therapeutic strategies. Nat Biotechnol 2006;24:15511557

3. Toke O. Antimicrobial peptides: New candidates in the fight against bacterial infections. Biopolymers 2005;80:717-735

4. Marcos JF, Muñoz A, Pérez-Payá E, Misra S, López-García B. Identification and rational design of novel antimicrobial peptides for plant protection. Annu Rev Phytopathol 2008;46:273-301

5. Montesinos E. Antimicrobial peptides and plant disease control. FEMS Microbiol Lett 2007;270:1-11

6. Papagianni M. Ribosomally synthesized peptides with antimicrobial properties: Biosynthesis, structure, function, and applications. Biotechnol Adv 2003;21:465-499

7. Rydlo T, Miltz J, Mor A. Eukaryotic antimicrobial peptides: Promises and premises in food safety. J Food Sci 2006;71:R125-R135

8. Brown KL, Hancock REW. Cationic host defense (antimicrobial) peptides. Curr Opin Immunol 2006;18:24-30

9. Braff MH, Hawkins MA, Di Nardo A, López-García B, Howell MD, Wong C, Lin K, Streib JE, Dorschner R, Leung DYM, Gallo RL. Structurefunction relationships among human cathelicidin peptides: Dissociation of antimicrobial properties from host immunostimulatory activities. J Immunol 2005;174:4271-4278

- This work characterizes the role of human cathelicidin LL37 on immune defense, indicating that antimicrobial activity against microbes resides within specific domains/motifs of the peptide, which do not directly correlate with immunomodulatory functions

10. Selsted ME, Ouellette AJ. Mammalian defensins in the antimicrobial immune response. Nat Immunol 2005;6:551-557

11. Brogden KA. Antimicrobial peptides: Pore formers or metabolic inhibitors in bacteria? Nat Rev Microbiol 2005;3:238-250

12. Yeaman MR, Yount NY. Mechanisms of antimicrobial peptide action and resistance. Pharmacol Rev 2003;55:27-55 
13. Jenssen $H$, Hamill $P$, Hancock REW. Peptide antimicrobial agents. Clin Microbiol Rev 2006;19:491-511

14. Casteels $P$, Tempst $P$. Apidaecin-type peptide antibiotics function through a non-poreforming mechanism involving stereospecificity. Biochem Biophys Res Commun 1994;199:339-345

15. Steffen H, Rieg S, Wiedemann I, Kalbacher H, Deeg A, Sahl HG, Peschel A, Gotz F, Garbe C, Schittek B. Naturally processed dermcidinderived peptides do not permeabilize bacterial membranes and kill microorganisms irrespective of their charge. Antimicrob Agents Chemother 2006;50:2608-2620

16. Vylkova S, Nayyar N, Li WS, Edgerton M. Human beta-defensins kill Candida albicans in an energy-dependent and salt-sensitive manner without causing membrane disruption. Antimicrob Agents Chemother 2007;51:154-161

17. Mochon AB, Liu HP. The antimicrobial peptide Histatin-5 causes a spatially restricted disruption on the Candida albicans surface, allowing rapid entry of the peptide into the cytoplasm. PLoS Pathog 2008;4:e1000190

- A detailed description of the non-lytic mode of action for Histatin 5 by using fluorescent labeling and confocal microscopic techniques that show a sequential differential peptide targeting to different cell locations and effects, depending on peptide concentration and time of exposure.

18. Monk BC, Niimi K, Lin S, Knight A, Kardos TB, Cannon RD, Parshot R, King A, Lun D, Harding DRK. Surface-active fungicidal D-peptide inhibitors of the plasma membrane proton pump that block azole resistance. Antimicrob Agents Chemother 2005;49:57-70

19. Muñoz A, López-García B, Marcos JF. Studies on the mode of action of the antifungal hexapeptide PAF26. Antimicrob Agents Chemother 2006;50:3847-3855

20. Hale JD, Hancock RE. Alternative mechanisms of action of cationic antimicrobial peptides on bacteria. Expert Rev Anti Infect Ther 2007;5:951-959

21. Otvos LJr. Antibacterial peptides and proteins with multiple cellular targets. J Pept Sci 2005;11:697-706

22. Aerts AM, Francois IEJA, Cammue BPA, Thevissen K. The mode of antifungal action of plant, insect and human defensins. Cell Mol Life Sci 2008;65:2069-2079

23. Peschel A, Sahl HG. The co-evolution of host cationic antimicrobial peptides and microbial resistance. Nat Rev Microbiol 2006;4:529-536 
24. Wade D, Boman A, Wahlin B, Drain CM, Andreu D, Boman HG, Merrifield RB. All-D amino acid-containing channel-forming antibiotic peptides. Proc Natl Acad Sci USA 1990;87:4761-4765

25. Luque-Ortega JR, Van't Hof W, Veerman ECl, Saugar JM, Rivas L. Human antimicrobial peptide histatin 5 is a cell-penetrating peptide targeting mitochondrial ATP synthesis in Leishmania. FASEB J 2008;22:1817-1828

26. Bulet P, Urge L, Ohresser S, Hetru C, Otvos L. Enlarged scale chemical synthesis and range of activity of drosocin, an O-glycosylated antibacterial peptide of Drosophila. Eur J Biochem 1996;238:64-69

27. Piers KL, Brown MH, Hancock REW. Improvement of outer membranepermeabilizing and lipopolysaccharide-binding activities of an antimicrobial cationic peptide by C-terminal modification. Antimicrob Agents Chemother 1994;38:2311-2316

28. Farnaud S, Spiller C, Moriarty L, Patel A, Gant V, Odell EW, Evans R. Interactions of lactoferricin-derived peptides with LPS and antimicrobial activity. FEMS Microbiol Lett 2004;233:193-199

29. Peschel A, Otto M, Jack RW, Kalbacher H, Jung G, Gotz F. Inactivation of the dit operon in Staphylococcus aureus confers sensitivity to defensins, protegrins, and other antimicrobial peptides. J Biol Chem 1999;274:8405-8410

30. Guo L, Lim KB, Poduje CM, Daniel M, Gunn JS, Hackett M, Miller SI. Lipid $A$ acylation and bacterial resistance against vertebrate antimicrobial peptides. Cell 1998;95:189-198

31. Peschel A, Jack RW, Otto M, Collins LV, Staubitz P, Nicholson G, Kalbacher H, Nieuwenhuizen WF, Jung G, Tarkowski A, van Kessel KPM, van Strijp JAG. Staphylococcus aureus resistance to human defensins and evasion of neutrophil killing via the novel virulence factor MprF is based on modification of membrane lipids with L-lysine. J Exp Med 2001;193:1067-1076

32. Papo N, Shai Y. A molecular mechanism for lipopolysaccharide protection of gram-negative bacteria from antimicrobial peptides. J Biol Chem 2005;280:10378-10387

33. Rosenfeld $Y$, Barra D, Simmaco M, Shai $Y$, Mangoni ML. A synergism between temporins toward gram-negative bacteria overcomes resistance imposed by the lipopolysaccharide protective layer. J Biol Chem 2006;281:28565-28574

34. Hsu STD, Breukink E, Tischenko E, Lutters MAG, de Kruijff B, Kaptein R, Bonvin AMJJ, van Nuland NAJ. The nisin-lipid II complex reveals a pyrophosphate cage that provides a blueprint for novel antibiotics. Nat Struct Mol Biol 2004;11:963-967 
- A detailed presentation of the structural characterization of the interaction of Nisin with its docking partner: the bacterial wall precursor Lipid II.

35. Wiedemann I, Breukink E, van Kraaij C, Kuipers OP, Bierbaum G, de Kruijff B, Sahl HA. Specific binding of nisin to the peptidoglycan precursor lipid II combines pore formation and inhibition of cell wall biosynthesis for potent antibiotic activity. J Biol Chem 2001;276:17721779

- A reference study that demonstrates multiple killing mechanisms of the model AMP Nisin, in permeating microbial cells but also blocking envelope peptidoglycan synthesis.

36. Breukink E, Wiedemann I, van Kraaij C, Kuipers OP, Sahl HG, de Kruijff $B$. Use of the cell wall precursor lipid II by a pore-forming peptide antibiotic. Science 1999;286:2361-2364

37. Dielbandhoesing SK, Zhang $\mathrm{H}$, Caro LH, van der Vaart JM, Klis FM, Verrips CT, Brul S. Specific cell wall proteins confer resistance to Nisin upon yeast cells. Appl Environ Microbiol 1998;64:4047-4052

38. Breukink E, de Kruijff B. Lipid II as a target for antibiotics. Nat Rev Drug Discov 2006;5:321-323

39. Brotz H, Bierbaum G, Leopold K, Reynolds PE, Sahl HG. The lantibiotic mersacidin inhibits peptidoglycan synthesis by targeting lipid II.

Antimicrob Agents Chemother 1998;42:154-160

40. Thevissen K, Idkowiak-Baldys J, Im YJ, Takemoto J, François IEJA, Ferket KKA, Aerts AM, Meert EMK, Winderickx J, Roosen J, Cammue BPA. SKN1, a novel plant defensin-sensitivity gene in Saccharomyces cerevisiae, is implicated in sphingolipid biosynthesis. FEBS Lett 2005;579:1973-1977

41. Stock SD, Hama H, Radding JA, Young DA, Takemoto JY. Syringomycin E inhibition of Saccharomyces cerevisiae: Requirement for biosynthesis of sphingolipids with very-long-chain fatty acids and mannose- and phosphoinositol-containing head groups. Antimicrob Agents Chemother 2000;44:1174-1180

42. Thevissen K, Cammue BPA, Lemaire K, Winderickx J, Dickson RC, Lester RL, Ferket KK, Van Even F, Parret AH, Broekaert WF. A gene encoding a sphingolipid biosynthesis enzyme determines the sensitivity of Saccharomyces cerevisiae to an antifungal plant defensin from dahlia (Dahlia merckii). Proc Natl Acad Sci USA 2000;97:9531-9536

43. Ramamoorthy V, Cahoon EB, Jia L, Thokala M, Minto RE, Shah DM. Glucosylceramide synthase is essential for alfalfa defensin-mediated growth inhibition but not for pathogenicity of Fusarium graminearum. Mol Microbiol 2007;66:771-786 
- This work demonstrates the importance of the gene GCS1, involved in glucosylceramide biosynthesis, for the sensitivity to the antifungal defensin MsDef1, but strikingly not to the related MtDef4.

44. Thevissen K, Warnecke DC, François IEJA, Leipelt M, Heinz E, Ott C, Zahringer U, Thomma BPHJ, Ferkel KKA, Cammue BPA. Defensins from insects and plants interact with fungal glucosylceramides. J Biol Chem 2004;279:3900-3905

45. Vylkova S, Li XS, Berner JC, Edgerton M. Distinct antifungal mechanisms: $\beta$-Defensins require Candida albicans Ssa1 protein, while Trk1p mediates activity of cysteine-free cationic peptides. Antimicrob Agents Chemother 2006;50:324-331

46. Lobo DS, Pereira IB, Fragel-Madeira L, Medeiros LN, Cabral LM, Faria J, Bellio M, Campos RC, Linden R, Kurtenbach E. Antifungal Pisum sativum defensin 1 interacts with Neurospora crassa cyclin F related to the cell cycle. Biochemistry 2007;46:987-996

- This work demonstrates the interaction of the plant defensin PSD1 with nuclear Cyclin F, its targeting to fungal nucleus, and indications that it might alter cell cycle progression, by using distinct experimental approaches. It is a significant demonstration of AMP specific nuclear targets in fungi.

47. Ibeas JI, Lee H, Damsz B, Prasad DT, Pardo JM, Hasegawa PM, Bressan RA, Narasimhan ML. Fungal cell wall phosphomannans facilitate the toxic activity of a plant PR-5 protein. Plant J 2000;23:375383

48. Hagen $S$, Marx F, Ram AF, Meyer $V$. The antifungal protein AFP from Aspergillus giganteus inhibits chitin synthesis in sensitive fungi. Appl Environ Microbiol 2007;73:2128-2134

49. Fujimura M, Ideguchi M, Minami Y, Watanabe K, Tadera K. Amino acid sequence and antimicrobial activity of chitin-binding peptides, Pp-AMP 1 and Pp-AMP 2, from Japanese bamboo shoots (Phyllostachys pubescens). Biosci Biotechnol Biochem 2005;69:642-645

50. Georgopapadakou NH. Update on antifungals targeted to the cell wall: Focus on $\beta-1,3$-glucan synthase inhibitors. Expert Opin Investig Drugs $2001 ; 10: 269-280$

51. Thevissen K, François IEJA, Aerts AM, Cammue BPA. Fungal sphingolipids as targets for the development of selective antifungal therapeutics. Curr Drug Targets 2005;6:923-928

52. Odds FC, Brown AJP, Gow NAR. Antifungal agents: mechanisms of action. Trends Microbiol 2003;11:272-279

53. Selitrennikoff $C P$, Nakata $M$. New cell wall targets for antifungal drugs. Curr Opin Investig Drugs 2003;4:200-205 
54. Heinisch J. Baker's yeast as a tool for the development of antifungal drugs which target cell integrity - an update. Expert Opin Drug Discov 2008;3:931-943

55. Yun DJ, Zhao Y, Pardo JM, Narasimhan ML, Damsz B, Lee H, Abad LR, Durzo MP, Hasegawa PM, Bressan RA. Stress proteins on the yeast cell surface determine resistance to osmotin, a plant antifungal protein. Proc Natl Acad Sci USA 1997;94:7082-7087

56. Li XS, Reddy MS, Baev D, Edgerton M. Candida albicans Ssa1/2p Is the cell envelope binding protein for human salivary Histatin 5. J Biol Chem 2003;278:28553-28561

57. Jang WS, Li XWS, Sun JNN, Edgerton M. The P-113 fragment of Histatin 5 requires a specific peptide sequence for intracellular translocation in Candida albicans, which is independent of cell wall binding. Antimicrob Agents Chemother 2008;52:497-504

- By using peptide fragments and sequence analogs the authors demonstrate the separation in the AMP histatin 5 of the binding cell wall protein Ssa2 from translocation into the cytosol, thus dissociating two necessary steps in the peptide interaction with target microorganisms.

58. Sun JNN, Li WS, Jang WS, Nayyar N, Sutton MD, Edgerton M. Uptake of the antifungal cationic peptide Histatin 5 by Candida albicans Ssa2p requires binding to non-conventional sites within the ATPase domain. Mol Microbiol 2008;70:1246-1260

- A remarkable characterization and mapping to a limited protein domain of the binding of the yeast Ssa2 HSP to the AMP Histatin 5, which is required for efficient cell uptake.

59. Li M, Lai YP, Villaruz AE, Cha DJ, Sturdevant DE, Otto M. Gram-positive three-component antimicrobial peptide-sensing system. Proc Natl Acad Sci USA 2007;104:9469-9474

- This work extends to Gram-positive bacteria the involvement of (threecomponent) sensor systems to signal and control the response to AMP, thus highlighting the existence of common signaling mechanisms along distinct microbial organism.

60. Hong RW, Shchepetov M, Weiser JN, Axelsen PH. Transcriptional profile of the Escherichia coli response to the antimicrobial insect peptide Cecropin A. Antimicrob Agents Chemother 2003;47:1-6

61. Tomasinsig L, Scocchi M, Mettulio R, Zanetti M. Genome-wide transcriptional profiling of the Escherichia coli response to a proline-rich antimicrobial peptide. Antimicrob Agents Chemother 2004;48:3260-3267

- Characterization of the bacterial global transcriptional response to a specific AMP. The expression changes of selected genes to a sequence 
derivative that is devoid of antimicrobial activity and to other unrelated AMP were also studied, and indicate distinctive responses.

62. Gamberi T, Cavalieri D, Magherini F, Mangoni ML, De Filippo C, Borro M, Gentile G, Simmaco M, Modesti A. An integrated analysis of the effects of Esculentin 1-21 on Saccharomyces cerevisiae. Biochim Biophys Acta 2007;1774:688-700

63. Vylkova S, Jang WS, Li WS, Nayyar N, Edgerton M. Histatin 5 initiates osmotic stress response in Candida albicans via activation of the Hog1 mitogen-activated protein kinase pathway. Eukaryot Cell 2007;6:18761888

64. Morton CO, Hayes A, Wilson M, Rash BM, Oliver SG, Coote P. Global phenotype screening and transcript analysis outlines the inhibitory mode(s) of action of two amphibian-derived, alpha-helical, cationic peptides on Saccharomyces cerevisiae. Antimicrob Agents Chemother 2007;51:3948-3959

- A reference study to determine the mechanism of action of antifungal peptides by using distinct/complementary functional genomic approaches. In addition, two different peptides were compared. Transcriptomic data and analysis of a genome-wide collection of yeast strains with gene deletions indicated that Magainin 2 and Dermaseptin S3 have both common and unique effects to fungal cells.

65. Bader MW, Sanowar S, Daley ME, Schneider AR, Cho US, Xu WQ, Klevit RE, Le Moual H, Miller S. Recognition of antimicrobial peptides by a bacterial sensor kinase. Cell 2005;122:461-472

- This work reveals an acidic surface domain from the PhoQ sensor kinase that participates in cationic antimicrobial peptide binding, as a first step in signal transduction across the bacterial membrane.

66. Fields PI, Groisman EA, Heffron F. A Salmonella locus that controls resistance to microbicidal proteins from phagocytic cells. Science 1989;243:1059-1062

67. Miller SI, Pulkkinen WS, Selsted ME, Mekalanos JJ. Characterization of defensin resistance phenotypes associated with mutations in the phoP virulence regulon of Salmonella typhimurium. Infect Immun 1990;58:3706-3710

68. McPhee JB, Lewenza S, Hancock REW. Cationic antimicrobial peptides activate a two-component regulatory system, PmrA-PmrB, that regulates resistance to polymyxin $B$ and cationic antimicrobial peptides in Pseudomonas aeruginosa. Mol Microbiol 2003;50:205-217

- Identification of an additional two-component system in Gram-negative bacteria (PmrAB) that regulates resistance to AMP. 
69. Gunn JS, Lim KB, Krueger J, Kim K, Guo L, Hackett M, Miller SI. PmrAPmrB-regulated genes necessary for 4-aminoarabinose lipid A modification and polymyxin resistance. Mol Microbiol 1998;27:1171-1182

70. Narasimhan ML, Coca MA, Jin JB, Yamauchi T, Ito Y, Kadowaki T, Kim KK, Pardo JM, Damsz B, Hasegawa PM, Yun DJ, Bressan RA. Osmotin is a homolog of mammalian adiponectin and controls apoptosis in yeast through a homolog of mammalian adiponectin receptor. Mol Cell 2005;17:171-180

71. Narasimhan ML, Damsz B, Coca MA, Ibeas JI, Yun DJ, Pardo JM, Hasegawa PM, Bressan RA. A plant defense response effector induces microbial apoptosis. Mol Cell 2001;8:921-930

72. Koo JC, Lee B, Young ME, Koo SC, Cooper JA, Baek D, Lim CO, Lee SY, Yun DJ, Cho MJ. Pn-AMP1, a plant defense protein, induces actin depolarization in yeasts. Plant Cell Physiol 2004;45:1669-1680

73. Ramamoorthy V, Zhao XH, Snyder AK, Xu JR, Shah DM. Two mitogenactivated protein kinase signalling cascades mediate basal resistance to antifungal plant defensins in Fusarium graminearum. Cell Microbiol 2007;9:1491-1506

- The authors extend here on the differential requirements to respond to the two related plant defensins MsDef1 and MtDef4, with an emphasis on the cell-signaling mediated by protein kinases cascades.

74. Morton CO, dos Santos SC, Coote P. An amphibian-derived, cationic, alpha-helical antimicrobial peptide kills yeast by caspase-independent but AIF-dependent programmed cell death. Mol Microbiol 2007;65:494507

- A detailed study that combines molecular genetics and cell biology methods to show that a specific AMP induces cell suicide/apoptosis in yeast

75. Andrés MT, Viejo-Díaz M, Fierro JF. Human lactoferrin induces apoptosis-like cell death in Candida albicans: Critical role of $\mathrm{K}^{+}$channelmediated $\mathrm{K}^{+}$efflux. Antimicrob Agents Chemother 2008;52:4081-4088

76. Leiter E, Szappanos H, Oberparleiter C, Kaiserer L, Csernoch L, Pusztahelyi T, Emri T, Posci I, Salvenmoser W, Marx F. Antifungal protein PAF severely affects the integrity of the plasma membrane of Aspergillus nidulans and induces an apoptosis-like phenotype. Antimicrob Agents Chemother 2005;49:2445-2453

77. Madeo F, Herker E, Wissing S, Jungwirth H, Eisenberg T, Frohlich KU. Apoptosis in yeast. Curr Opin Microbiol 2004;7:655-660

78. Lupetti A, Paulusma-Annema A, Senesi S, Campa M, van Dissel JT, Nibbering $\mathrm{PH}$. Internal thiols and reactive oxygen species in candidacidal 
activity exerted by an N-terminal peptide of human lactoferrin. Antimicrob Agents Chemother 2002;46:1634-1639

79. Aerts AM, François IEJA, Meert EMK, Li QT, Cammue BPA, Thevissen $\mathrm{K}$. The antifungal activity of RsAFP2, a plant defensin from Raphanus sativus, involves the induction of reactive oxygen species in Candida albicans. J Mol Microbiol Biotechnol 2007;13:243-247

80. Helmerhorst EJ, Troxler RF, Oppenheim FG. The human salivary peptide histatin 5 exerts its antifungal activity through the formation of reactive oxygen species. Proc Natl Acad Sci USA 2001;98:14637-14642

81. Veerman ECI, Nazmi K, van Hof W, Bolscher JGM, den Hertog AL, Amerongen AVN. Reactive oxygen species play no role in the candidacidal activity of the salivary antimicrobial peptide histatin 5 . Biochem J 2004;381:447-452

82. Castle M, Nazarian A, Yi SS, Tempst $P$. Lethal effects of apidaecin on Escherichia coli involve sequential molecular interactions with diverse targets. J Biol Chem 1999;274:32555-32564

83. Park CB, Yi KS, Matsuzaki K, Kim MS, Kim SC. Structure-activity analysis of buforin II, a histone $\mathrm{H} 2 \mathrm{~A}$-derived antimicrobial peptide: The proline hinge is responsible for the cell-penetrating ability of buforin II. Proc Natl Acad Sci USA 2000;97:8245-8250

- A study that highlights the potential of using AMP sequence analogs with specific amino acid residue substitutions to characterize the interaction of peptides with microbes. The work demonstrated that the proline hinge is a key structural factor for the cell-penetrating ability of peptide buforin II.

84. Kragol G, Hoffmann R, Chattergoon MA, Lovas S, Cudic M, Bulet P, Condie BA, Rosengren KJ, Montaner LJ, Otvos LJr. Identification of crucial residues for the antibacterial activity of the proline-rich peptide, pyrrhocoricin. Eur J Biochem 2002;269:4226-4237

85. Park CB, Kim HS, Kim SC. Mechanism of action of the antimicrobial peptide buforin II: Buforin II kills microorganisms by penetrating the cell membrane and inhibiting cellular functions. Biochem Biophys Res Commun 1998;244:253-257

86. van der Kraan MIA, van Marle J, Nazmi K, Groenink J, Van't Hof W, Veerman ECI, Bolscher JGM, Arnerongen AVN. Ultrastructural effects of antimicrobial peptides from bovine lactoferrin on the membranes of Candida albicans and Escherichia coli. Peptides 2005;26:1537-1542

87. van der Weerden NL, Lay FT, Anderson MA. The plant defensin, NaD1, enters the cytoplasm of Fusarium oxysporum hyphae. J Biol Chem 2008;283:14445-14452 
88. Gyurko C. Killing of Candida albicans by histatin 5: Cellular uptake and energy requirement. Antonie Van Leeuwenhoek Int J Gen Molec Microbiol 2001;79:297-309

89. Powers JPS, Martin MM, Goosney DL, Hancock REW. The antimicrobial peptide polyphemusin localizes to the cytoplasm of Escherichia coli following treatment. Antimicrob Agents Chemother 2006;50:1522-1524

90. Gelhaus C, Jacobs T, Andra J, Leippe M. The antimicrobial peptide NK2 , the core region of mammalian NK-lysin, kills intraerythrocytic Plasmodium falciparum. Antimicrob Agents Chemother 2008;52:17131720

91. Haukland HH, Ulvatne H, Sandvik K, Vorland LH. The antimicrobial peptides lactoferricin $B$ and magainin 2 cross over the bacterial cytoplasmic membrane and reside in the cytoplasm. FEBS Lett 2001;508:389-393

92. Henriques ST, Melo MN, Castanho MARB. Cell-penetrating peptides and antimicrobial peptides: how different are they? Biochem J 2006;399:1-7

93. Foged C, Nielsen HM. Cell-penetrating peptides for drug delivery across membrane barriers. Expert Opin Drug Deliv 2008;5:105-117

94. Palm C, Netzerea S, Hallbrink M. Quantitatively determined uptake of cell-penetrating peptides in non-mammalian cells with an evaluation of degradation and antimicrobial effects. Peptides 2006;27:1710-1716

95. Jung HJ, Park Y, Hahm KS, Lee DG. Biological activity of Tat (47-58) peptide on human pathogenic fungi. Biochem Biophys Res Commun 2006;345:222-228

96. Jones AT. Gateways and tools for drug delivery: Endocytic pathways and the cellular dynamics of cell penetrating peptides. Int J Pharm 2008;354:34-38

97. Duchardt F, Fotin-Mleczek M, Schwarz H, Fischer R, Brock R. A comprehensive model for the cellular uptake of cationic cell-penetrating peptides. Traffic 2007;8:848-866

98. Console S, Marty C, García-Echeverría C, Schwendener R, BallmerHofer K. Antennapedia and HIV transactivator of transcription (TAT) "protein transduction domains" promote endocytosis of high molecular weight cargo upon binding to cell surface glycosaminoglycans. J Biol Chem 2003;278:35109-35114

99. Subbalakshmi C, Sitaram N. Mechanism of antimicrobial action of indolicidin. FEMS Microbiol Lett 1998;160:91-96

100. Patrzykat A, Friedrich CL, Zhang LJ, Mendoza V, Hancock REW. Sublethal concentrations of pleurocidin-derived antimicrobial peptides 
inhibit macromolecular synthesis in Escherichia coli. Antimicrob Agents Chemother 2002;46:605-614

101. Friedrich CL, Rozek A, Patrzykat A, Hancock REW. Structure and mechanism of action of an indolicidin peptide derivative with improved activity against gram-positive bacteria. J Biol Chem 2001;276:2401524022

102. Lehrer RI, Barton A, Daher KA, Harwig SS, Ganz T, Selsted ME. Interaction of human defensins with Escherichia coli. Mechanism of bactericidal activity. J Clin Invest 1989;84:553-561

103. Boman HG, Agerberth B, Boman A. Mechanisms of action on Escherichia coli of cecropin P1 and PR-39, two antibacterial peptides from pig intestine. Infect Immun 1993;61:2978-2984

104. Kragol G, Lovas S, Varadi G, Condie BA, Hoffmann R, Otvos LJr. The antibacterial peptide pyrrhocoricin inhibits the ATPase actions of DnaK and prevents chaperone-assisted protein folding. Biochemistry 2001;40:3016-3026

- The initial characterization of the binding of pyrrhocoricin to Escherichia coli heat shock protein DnaK, that results in the impairment of the protein activity and thus

105. Otvos LJr, O I, Rogers ME, Consolvo PJ, Condie BA, Lovas S, Bulet P, Blaszczyk-Thurin M. Interaction between heat shock proteins and antimicrobial peptides. Biochemistry 2000;39:14150-14159

106. Kramer NE, Van Hijum SAFT, Knol J, Kok J, Kuipers OP. Transcriptome analysis reveals mechanisms by which Lactococcus lactis acquires nisin resistance. Antimicrob Agents Chemother 2006;50:1753-1761

- A unique example of an alternative approach to the characterization of the bacterial resistance to AMP, by using transcriptomic approaches. The authors compared the global gene expression profile of a bacterial strain resistant to Nisin with that of the parental strain. A set of general responses is proposed.

107. Boix E, Nogues MV. Mammalian antimicrobial proteins and peptides: overview on the RNase A superfamily members involved in innate host defence. Mol Biosyst 2007;3:317-335

108. Chadha $\mathrm{P}$, Das $\mathrm{RH}$. A pathogenesis related protein, AhPR10 from peanut: an insight of its mode of antifungal activity. Planta 2006;225:213222

- Demonstration of the linkage between the RNase activity of a plant PR10 and its antimicrobial properties; these latter require, although are not linked to, internalization into fungal cells. 
109. Nogales J, Muñoz S, Olivares J, Sanjuán J. Sinorhizobium meliloti genes involved in tolerance to the antimicrobial peptide protamine. FEMS Microbiol Lett 2006;264:160-167

110. Stephens C, Harrison SJ, Kazan K, Smith FWN, Goulter KC, Maclean DJ, Manners JM. Altered fungal sensitivity to a plant antimicrobial peptide through over-expression of yeast cDNAs. Curr Genet 2005;47:194-201

111. Matsuzaki K, Sugishita K, Miyajima K. Interactions of an antimicrobial peptide, magainin 2, with lipopolysaccharide-containing liposomes as a model for outer membranes of gram-negative bacteria. FEBS Lett 1999;449:221-224

112. Hsu CH, Chen CP, Jou ML, Lee AYL, Lin YC, Yu YP, Huang WT, Wu $\mathrm{SH}$. Structural and DNA-binding studies on the bovine antimicrobial peptide, indolicidin: evidence for multiple conformations involved in binding to membranes and DNA. Nucleic Acids Res 2005;33:4053-4064

113. Ulvatne $\mathrm{H}$, Samuelsen $\mathrm{O}$, Haukland $\mathrm{HH}$, Kramer M, Vorland LH. Lactoferricin B inhibits bacterial macromolecular synthesis in Escherichia coli and Bacillus subtilis. FEMS Microbiol Lett 2004;237:377-384 
Figure 1

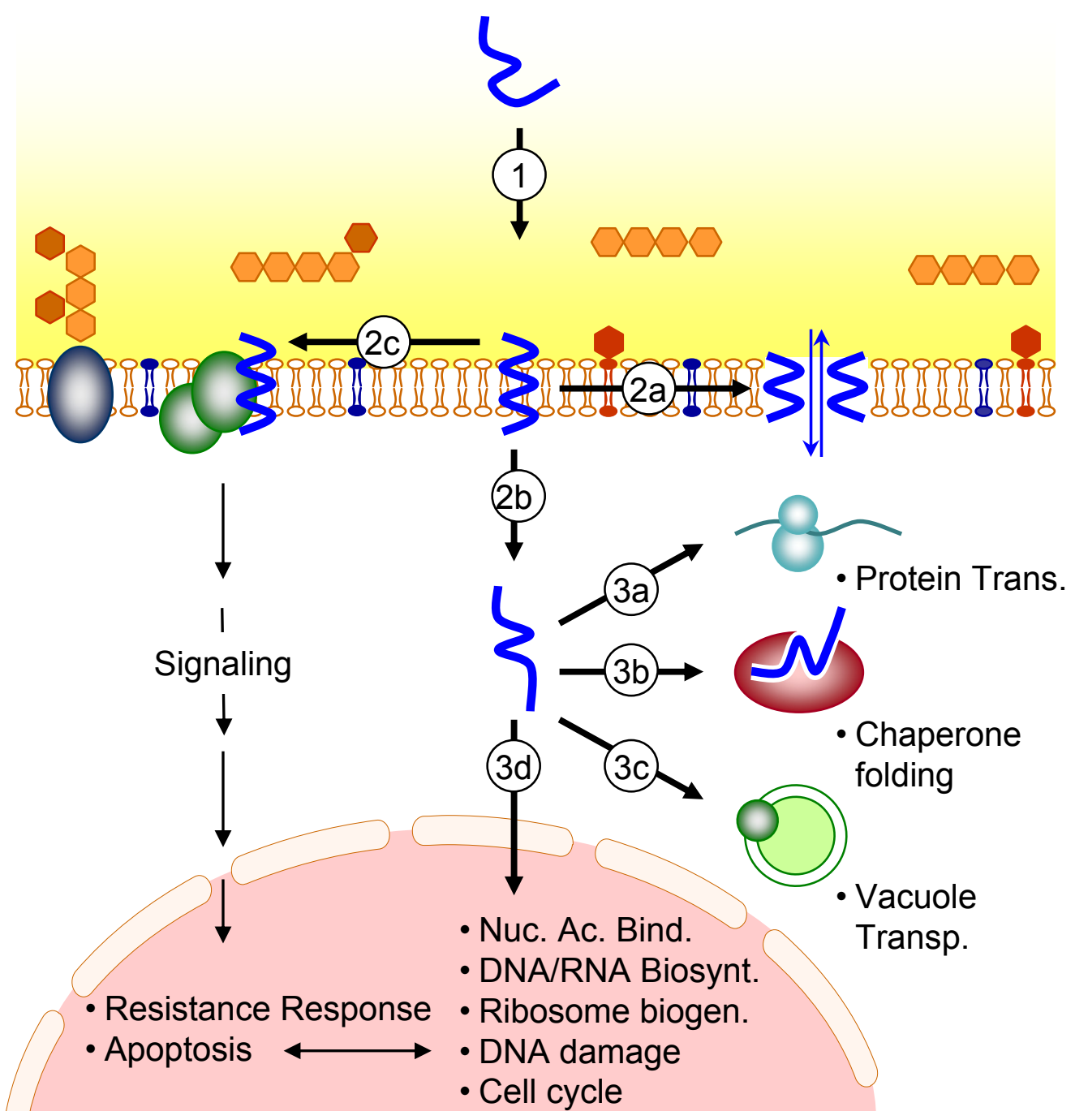

